\title{
Molecular neuro-biological and systemic health benefits of achieving dopamine homeostasis in the face of a catastrophic pandemic (COVID- 19): A mechanistic exploration
}

Downs BW ${ }^{1}$, Blum K ${ }^{1-6^{*}}$, Bagchi $\mathrm{D}^{1,7}$, Kushner $\mathrm{S}^{8}$, Bagchi M${ }^{9}$, Galvin $\mathrm{JM}^{10}$, Lewis $\mathrm{MCG}^{11}$, Siwicki $\mathrm{D}^{6}$, Brewer R $\mathrm{B}^{6}$, Boyett $\mathrm{B}^{3}$, Baron $\mathrm{D}^{2}$, Giordano $\mathrm{J}^{12}$ and Badgaiyan $\mathrm{RD}^{13}$

${ }^{1}$ Department of Nutrigenomics Research, Victory Nutrition International, Inc., Lederach, PA, USA

${ }^{2}$ Western University, Health Sciences, Graduate School of Biomedical Sciences, Pomona, CA, USA

${ }^{3}$ Division of Neuroscience and Addiction Research, Pathway Healthcare, Birmingham, AL, USA

${ }^{4}$ Eotvos Loránd University, Institute of Psychology, Budapest, Hungary

${ }^{5}$ Department of Psychiatry, Wright State University Boonshoft School of Medicine and Dayton VA Medical Center, Dayton, OH, USA

${ }^{6}$ Division of Precision Nutrition, GARS IP., LLC, Hollywood Fl., USA, \& Geneus Health, LLC., San Antonio, TX, USA

${ }^{7}$ Department of Pharmacological \& Pharmaceutical Sciences, University of Houston College of Pharmacy, Houston, TX, USA

${ }^{8}$ ALM Research \& Development, Oldsmar, FL, USA

${ }^{9}$ Dr. Herbs LLC, Concord, CA, USA

${ }^{10}$ Vitality Medical Wellness Institute, PLLC, Charlotte, NC, USA

${ }^{11}$ Departments of Anatomy \& Psychiatry, Howard University, School of Medicine, Washington, D., USA

${ }^{12}$ National Institute of Holistic and Addiction Studies, Davie, FL, USA

${ }^{13}$ Department of Psychiatry, ICHAN School of Medicine, Mount Sinai, New York, NYC. \& Department of Psychiatry, South Texas Veteran Health Care System, Audie L. Murphy Memorial VA Hospital, San Antonio, TX, Long School of Medicine, University of Texas Medical Center, San Antonio, TX, USA

\begin{abstract}
In the face of the global pandemic of COVID 19, approaching 1.75 Million infected worldwide (4/12/2020) and associated mortality (over 108, 000 as of 4/12/2020) as well-as other catastrophic events including the opioid crisis, a focus on brain health seems prudent [1] (https://www.coronavirus.gov). This manuscript reports on the systemic benefits of restoring and achieving dopamine homeostasis to reverse and normalize thoughts and behaviors of Reward Deficiency Syndrome (RDS) dysfunctional conditions and their effects on behavioral physiology; function of reward genes; and focuses on digestive, immune, eye health, and the constellation of symptomatic behaviors. The role of nutrigenomic interventions on restoring normal brain functions and its benefits on these systems will be discussed. We demonstrate that modulation of dopamine homeostasis using nutrigenomic dopamine agonists, instead of pharmaceutical interventions, is achievable. The allied interlinking with diverse chronic diseases and disorders, roles of free radicals and incidence of anaerobic events have been extensively highlighted. In conjunction, the role of dopamine in aspects of sleep, rapid eye movement and waking are extensively discussed. The integral aspects of food indulgence, the influence of taste sensations, and gut-brain signaling are also discussed along with a special emphasis on ocular health. The detailed mechanistic insight of dopamine, immune competence and the allied aspects of autoimmune disorders are also highlighted. Finally, the integration of dopamine homeostasis utilizing a patented gene test and a research-validated nutrigenomic intervention are presented. Overall, a cutting-edge nutrigenomic intervention could prove to be a technological paradigm shift in our understanding of the extent to which achieving dopamine homeostasis will benefit overall health.
\end{abstract}

\section{Introduction: The proposal}

Since November/December of 2019, a series of patients with atypical pneumonia have been reported in Wuhan, China. On 12 January 2020, the World Health Organization (WHO) temporarily named this new virus as the 2019 novel coronavirus $(2019-\mathrm{nCoV})$ and on 11 February 2020, the disease caused by the 2019-nCoV was named coronavirus disease (COVID-19). The COVID-19 pandemic has spread all over the world. To make matters worse the USA is still facing over 185 deaths per day due to the opioid epidemic. Together these catastrophes not only caused hundreds of thousands of premature fatalities, but the entire globe is under enormous stress because currently there is no treatment for this disease. There is therefore a need for out of the box thinking $[2,3]$.

${ }^{*}$ Correspondence to: Kenneth Blum, Western University Health Sciences Graduate School of Biomedical Sciences, Pomona, CA, USA, E-mail: drd2gene@gmail.com

Key words: reward deficiency syndrome, dopamine, neurotrophic, anaerobic, hypoxic, dopamine homeostasis

Received: April 11, 2020; Accepted: April 28, 2020; Published: April 30, 2020 
It is well-known that many polymorphic genes, particularly the genetic determinants of hypodopaminergia, are associated with Substance Use Disorder (SUD). These genes are also associated with a predisposition to stress [4,5]. The National Institutes of Health (NIH) support neuroimaging research and molecular genetic applied technologies to improve understanding of the complex functions of brain reward circuitry that play crucial roles in addiction and stress symptomatology [6].

It is noteworthy that mice lacking adaptive immunity are significantly more susceptible to develop psychiatric symptoms like PTSD when compared with mice that have normal immune systems [7].

In this context it is worth mentioning that raising Endorphinergic function significantly increases the immune response. On the contrary, chronic administration of exogenous opioids significantly suppresses the immune response and increase vulnerability to infections. Moreover, exogenous opioids such as morphine and fentanyl have been found to impair the function of macrophages, natural killer cells and T-cells and to weaken the gut barrier in vitro and in animal studies. However, in contrast, immune cells, including neutrophils, macrophages and T-cells, have been shown to secrete endogenous opioid peptides such as endorphins, enkephalins and dynorphins, which then bind to peripheral opioid receptors to relieve inflammatory and neuropathic pain. In addition to cytokines, hormones and bacterial products, the release of opioid peptides is stimulated by the application of exogenous opioids [8]. Along these lines, Blum's work validated by neurochemical measurements, showed that the enkephalinase inhibitor D-Phenylalanine (DPA) not only increases brain endorphins in animal models but also reduces stress in humans $[9,10]$. Importantly, there are a large number of interactions at molecular and cellular levels between the nervous system and the immune system. Certainly, Janković \& Radulović, demonstrated that the opioid neuropentapeptide methionine-enkephalin (Met-Enk) is involved in humoral and cellmediated immune reactions [11]. Therefore, based on this previous research, one way to treat catastrophic stress is to enhance endorphin function by utilization of enkephalinase inhibitors like DPA.

Relevant genotype-phenotype relationships can be characterized using the Genetic Addiction Risk Score (GARS ) [12]. Based on GARS values, dopamine homeostasis may be achieved via customization of neuro-nutrient supplementation with particular emphasis on enhancement of immunological function [13-16]. Precision Behavioral Management (PBM), and putative pro-dopamine regulation should be accompanied by interventions like mindfulness and trauma therapy. Recognizing the immune system as a "sixth sense" may shift the paradigm in treating such disorders [17-21]. This out of the box thinking is hereby supported by the following commentary provided in this treatise.

\section{Understanding reward deficiency syndrome (RDS)}

It is well established that integrity of the neurotransmitter system is essential for normal homeostatic functioning of the brain. Discovering and understanding Reward Deficiency Syndrome (RDS) and defining how genetic factors underlying aberrant neurotransmission in RDS behaviors are influenced by lifestyle activities (i.e. epigenetics) has been the life's work of one of our authors (KB). The primary research objective has been identifying genetic predispositions that induce the expansive range of RDS dysfunctional conditions and behaviors, restoring dopamine homeostasis with nutrigenomic interventions, thereby restoring normal brain function, reward processing and the integrity of resting-state functional connectivity essential for healthy homeostatic functioning [22]. Discovery of the association of severe alcoholism with the Dopamine D2 Receptor TaqI A1 allele by Blum and Noble was published in JAMA in 1990 [23]. Continued efforts to challenge or refute this seminal discovery resulted in a veritable multitude of clinical studies over a 20 -year span, almost all of which unintentionally but overwhelmingly confirmed the validity of this paradigm-shifting discovery. Subsequently, on August 15, 2011, a culmination of this validation resulted in the American Society of Addiction Medicine (ASAM) publishing a change in the definition of addiction. This position statement was most recently confirmed again on September 15, 2019 [24]. The ASAM position statement established that addiction was no longer considered purely a psychological disorder. Instead, addiction was defined as " a treatable, chronic medical disease involving complex interactions among brain circuits, genetics, the environment, and an individual's life experiences." The consequence is an imbalance in the neurobiochemical 'hardwiring' of the brain; having at least a $50 \%$ etiological contribution by genetic predispositions in the brain's reward circuitry.

Reward Deficiency Syndrome (RDS) has emerged as a widely accepted condition with a constellation of symptomatic behaviors and manifestations that have also been published in the SAGE Encyclopedia of Abnormal \& Clinical Psychology 2017 [25]. A sampling of the expansive range of RDS conditions and behaviors is indicated in Table 1.

The notion of the authors has been that reducing cravings; restoring self-control, reward satisfaction, and mental sharpness; and redeeming happiness in life is best accomplished by achieving dopamine homeostasis with nutrigenomic dopamine agonists rather than by pharmaceutical interventions [22]. Many pharmaceutical therapies for RDS conditions are dopamine antagonists that can, in short term, simply induce pharmacological interference in neurotransmitter

Table 1. Different reward deficiency syndrome (RDS) behaviors: A function of reward genes

\begin{tabular}{|c|c|c|c|c|c|}
\hline \multicolumn{2}{|c|}{ Addictive Behaviors } & \multicolumn{2}{|c|}{ Impulsive Behaviors } & \multirow{2}{*}{$\begin{array}{c}\text { Obsessive Compulsive } \\
\text { Behaviors }\end{array}$} & \multirow[t]{2}{*}{ Personality Disorders } \\
\hline Substance related & Non substance related & Spectrum Disorders & Disruptive Impulsive & & \\
\hline Alcohol & Thrill Seeking (novelty) & $\mathrm{ADD} / \mathrm{ADHD}$ & Anti-Social & Body Dysmorphic & Paranoid \\
\hline Cannabis & Sexual Sadism & Tic Syndrosme & Conduct & Hoarding & Schizoid \\
\hline Opioids & Sexual Masochism & Tourette Syndrome & Intermittent Explosive & $\begin{array}{l}\text { Hair-Pulling (Trichotillo- } \\
\text { mania) }\end{array}$ & Borderline \\
\hline Sedatives/Hypnotics & Hyper-sexuality & Autism & Oppositional Defiant & Excoriation (Skin Picking) & Bipolar \& Depression \\
\hline stimulants & Gambling & Stuttering & Exhibitionism & $\begin{array}{c}\text { Non-suicidal Self-Injury } \\
\text { (mutilation) }\end{array}$ & $\begin{array}{c}\text { Anger \& Pathological } \\
\text { Violence }\end{array}$ \\
\hline Tobacco & Internet Gaming & & Kleptomania & Pathological Gambling & Narcissistic \\
\hline Glucose \& Fat & & & Pyromania & & Avoidant \\
\hline Food & & & & & Dependant \\
\hline
\end{tabular}


transactions and act as dopamine antagonists to manage symptoms to blunt unhealthy deviant RDS behaviors. Research has demonstrated that achieving dopamine homeostasis with nutrigenomic dopamine agonists provides more successful and sustainable benefits [22].

Most RDS behaviors can be categorized into one of the following categories of addictions, obsessions, compulsions, impulsions and personality disorders. Up to this time, attention has been primarily focused on the importance of the functional competence of neurotransmitters in the Brain Reward Cascade (BRC) and their influence on those behaviors, resulting in a plethora of clinical studies and published papers. By default, this intense focus of attention is the result of an epidemic of mental health, mood, pain and behavioral disorders leading to an epidemic of opioid prescriptions and addictions [26].

According to the National Alliance of Mental Health Disorders, 1 in 5 Americans will experience a mental health disorder [27]. This statistical reality translates to an enormous need for effective therapeutic interventions, which, under the current treatment protocols, results in a massive usage of prescribed opioids [28]. However, conventional pharmaceutical treatments, therapies, programs and fellowship programs (i.e. AA, Celebrate Recovery, etc.) do not seem to have consistently and/or significantly reduced recidivism rates nor blunted the incidence of opioid usage or rate of prescriptions [29].

\section{Chronic diseases, reactive oxygen species (ROS) and anaerobic events}

Considering the prevalence of diverse chronic disorders and diseases, it is not logical to assume that an individual experiencing a mental health/behavioral disorder is otherwise completely healthy. Impairments in the functional competence of the BRC, by default, are generally accompanied by impaired functions in various other tissues of the body and disorders including, diabetes, obesity, CVD, digestive disorders, arthritis and numerous other inflammatory dysfunctions. Neurotransmitters in the brain reward cascade (BRC) have a significant influence on human health and different organs, tissues, and cellular and subcellular tissues in the body. Therefore, other important salient features that contribute to functional impairments are significant therapeutic targets that merit serious investigation.

\section{Oxidative stress and free radicals - aka 'reactive oxygen species' (ROS)}

Reactive Oxygen Species (ROS) are produced primarily from 3 different sources: 1 . As by-products of cellular energy production; 2. from exposure to exogenous sources such as environmental pollutants; and 3. as a result of a loss in the ability of cells to effectively use available oxygen (contributed to by chronic nutrient deficiencies and exposure to environmental pollutants, etc.) that impair intracellular organelles' structures and functions. These events result in an increase in anaerobic/hypoxic events and pathologies. All chronic diseases are characterized by a significant increase in anaerobic (increased acidic/ hypoxic bio-environment) pathological events and a consequential reduction in the ability to effectively utilize available oxygen [30]. Excessive oxygen free radical-induced oxidative stress and subsequent damages to lipids, proteins and cellular macromolecules are prevalent factors that can significantly impair neuronal functioning and exert damaging effects on all biological tissues, enzymes and fluids. Such oxidative distress induces a greater demand on endogenous antioxidant enzymes, Ex.: SOD, GSH, Dehydrogenases, CYP 450 enzymes, etc. Research evaluating the damaging effect of oxidative agents on the functionality of neuronal brain cells and the ability to repair neuronal function with neurotrophic factors is of great interest.

Bagchi et al. have shown that dementia is an anaerobic pathology characterized by an increase in hydrogen peroxide, peroxyl radical, hypochlorite radical, hypochlorous acid, $\mathrm{OCl}+\mathrm{HOCl}$ and hydroxyl radical. Individually and collectively, these oxidative species can cause significant injury to the neuronal cells leading to neurological damage, neuromuscular injury and even fibrillation [31]. The key feature of these anaerobic, hypoxic, and increased acidic pathological events is a reduced ability to effectively use oxygen and water, which promotes a sequela of anaerobic and inflammatory events. Moreover, an increase in the anaerobic/hypoxic properties of tissues creates a suitable biological environment for the incubation and proliferation of anaerobic organisms and processes such as yeasts, worms, mold, bacteria (i.e. infections), inflammation, parasites, viruses, etc. that progresses to definable chronic degenerative pathological conditions. In fact, the evidence indicates that the herpes virus contributes to the etiology of chronic neurodegenerative disorders like Parkinson's Disease, Alzheimer's Disease, MS, ALS, Encephalitis, Meningitis, Mononucleosis, Epstein Barr Virus, cytomegalovirus, etc. [32].

Correcting the hypoxic state of brain tissues by improving Blood Oxygen Level Dependent signaling and functioning not only improves BRC neurotransmitter functioning and cross talk but reduces oxidative stress and damage to and symptoms of those anaerobic disorders. RDS conditions are also characterized by a reduced ability to achieve healthy Blood Oxygen Level Dependent functions and signaling in the brain, which are also anaerobic pathological events. Animal and human research confirm the benefits of improving Blood Oxygen Level Dependent signaling with a pro-dopaminergic agent [33]. In addition to improving and effectively managing reward circuitry and reward processing, reversing anaerobic sequela and promoting Blood Oxygen Level Dependent signaling and functionality also restores healthy aerobic metabolism, important for optimizing gene expression and systemic health. A clinical study demonstrated that the KB220Z, a nutrigenomic dopamine agonist, restores Blood Oxygen Level Dependent signaling and functionality and improved functional connectivity, or 'cross talk' in 10 abstinent heroin addicts [34]. Increased functional connectivity ('cross talk) was observed in a putative network that included the dorsal anterior cingulate, medial frontal gyrus, nucleus accumbens, posterior cingulate, occipital cortical areas and cerebellum in one hour after oral administration.

\section{Behavioral physiology: Sleep, rapid eye movement and waking}

It is well established that sleep is necessary and responsible for optimal functions required for systemic bio-homeostasis. Animal research in dopamine D2 Receptor (DRD2) knock out mice demonstrates that dopamine is important in sleep-wake regulation. However, the mice experienced an increased number of periodic awakenings and nonRapid Eye Movement (nREM) sleep. This indicates that dopamine plays some role in achieving sustainable sleep but a vital role in wakefulness [35]. Another study demonstrates that serotonin (5-HT) and dopamine (DA) are involved in promoting waking and inhibition of slow wave sleep (SWS) and/or rapid-eye-movement sleep (REMS). This research demonstrates that there is an upsurge of firing activity of DA neurons simultaneously with an increased release of DA in the ventral tegmental area (VTA) and allied forebrain structures during waking and REM Sleep. During waking, dopamine neurons, located in the ventral periaqueductal grey matter, induce an expression of FOS 
Downs BW (2020) Molecular neuro-biological and systemic health benefits of achieving dopamine homeostasis in the face of a catastrophic pandemic (COVID- 19): A mechanistic exploration

proteins, which have a critical function in regulating the development of cells destined to form and maintain the skeleton and are reported to have an important role in signal transduction, cell proliferation and differentiation. Confirming other research, these researchers conclude that dopamine neurons may participate in the promotion of waking [36].

Normal dopamine homeostasis is crucial for an optimal quality of life, especially for recovering addicts [36,37]. Sleep has also been linked with metabolic clearance of neurotoxins. It was also demonstrated in mice that natural sleep is associated with a $60 \%$ increase in interstitial space indicating an increase in convective exchange of cerebrospinal fluid with interstitial fluid. This process was shown to result in an increase in $\beta$-amyloid clearance during sleep [34,38]. Moreover, achieving dopamine homeostasis with a nutrigenomic dopamine agonist (KB220Z) has also been shown to facilitate complete amelioration of long-term terrifying lucid nightmares, also improving dopamine stability and functional connectivity between networks of the brain reward circuitry in both rodents and humans; resulting in happy dreams in humans [39].

It has been suggested that a dopaminergic deficit may be responsible for circadian rhythm dysregulation, which occurs during onset of neurodegeneration. Researchers have demonstrated that the contribution of slow brain oscillations including delta, theta, alpha, and sigma frequencies $(0.5-16 \mathrm{~Hz})$ to the sleep electroencephalography (EEG) is regulated by circadian and homeostatic influences and reflects functional aspects of wakefulness and sleep [40,41]. Thus, it was hypothesized that restitution of rapid eye movement (REM) sleep and an improvement in Parkinson disease's symptoms could be achieved with the regulated use of dopamine agonists during the night. A study was conducted in twenty Parkinson's patients over a period of one month following treatment with subcutaneous nocturnal administration of apomorphine, a dopamine receptor agonist, at the beginning of each REM stage. Based on the results of symptomatic benefits, the researcher concluded that sleep alteration in Parkinson's disease can be improved by stimulation of D2 receptors [42].

\section{Dopamine homeostasis and gastrointestinal health}

An example of the mechanistic effects of dopamine on colon function is observed in Parkinson's Disease (PD). In animal models, Garrido-Gil et al. found that a reduction in central dopamine levels caused a reduction in colonic dopamine types 1 and 2 receptor expression along with an increase in the colonic levels of dopamine and a reduction in the levels of acetylcholine. This is believed to contribute to a significant lowering in gut motility. An increase in the colonic levels of inflammatory and oxidative stress markers following depletion of central dopaminergic system, along with activation of the pro-inflammatory Renin Angiotensin System (RAS) was observed. The researchers also found that gut inflammation induced changes in nigrostriatal dopaminergic homeostasis, dopaminergic neuron death, and increased levels of nigral pro-inflammatory markers accompanied by RAS pro-inflammatory activity. The researchers concluded that dysregulation of the neural bidirectional gut-brain interaction may be responsible for the early gut disturbances observed in parkinsonian patients now considered as having a subtype of RDS. They also indicate that the increase in vulnerability of nigral dopaminergic neurons occurs after gut inflammation [43]. This research strongly suggests that effectively promoting dopamine synthesis, transport, reception, and degradation/reuptake could be an effective way to improve not only PD neurological symptoms but gut motility and function as well.

\section{Food intake}

\section{Taste sensors and gut-brain signaling}

Nutrient receptor 'chemosensors' in the gut have been the subject of extensive research to evaluate taste sensory properties. Nutrient appetite is influenced by oral sugar, fat, salt and savory taste receptors [44]. These receptors and other gut receptors are linked to digestive, metabolic, and satiating effects that influence nutrient utilization and inhibit appetite. Furthermore, gut chemosensors provide positive feedback signals that condition food preferences and stimulate appetite. It has been well demonstrated that postoral sensors (taste sensors situated in the posterior of the mouth) influence carbohydrate and fat responses in the brain dopamine reward system [45]. This research reveals that chemosensors are one of the mechanisms that initiate food preference learning, stimulate appetite and reward signaling and feedback, and contributes to our understanding of the relationship of both neural and hormonal gut-brain signaling pathways.

\section{Vision and eye health}

An increase in dopamine release has been demonstrated during light adaptation and is accompanied by a suppression of rod signaling in light-adapted retinas. Current studies have demonstrated that dopamine, mediating through DAD1 receptors, could potentially induce a sensitization of the rod pathway in dim light conditions through GABA type C receptors [46]. Vision has been shown to adapt to the extensive changes in light intensity occurring over a period of 24 hours. This unique adaptation process is highly dependent on the rhythms in cellular and molecular processes to synchronize the pathways linking the cell-specific molecular machineries to their cognate outputs. The process is orchestrated by a network of circadian clocks located within the retina of the eye that recognize changes in light and respond accordingly, which are regulated by genes in the retina. The circadian clocks are synchronized to respond to day/night cycles along with simultaneous fine-tune detection and processing of light information that occur over a 24-hour period. This recognition and response signaling process is what regulates retinal homeostasis. It is known that both melatonin and dopamine participate in a regulatory role in this physiological process, being both outputs and inputs for clocks. This research provides insight into a novel mechanistic intervention that reveals a potential role for dopamine and melatonin in resolving retinal degeneration [47]. However, while regulating melatonin is important, health care professionals must be cognizant that increased levels of brain melatonin can lead to increased alcohol intake [48].

\section{Role of dopamine in immune competence}

Psychosocial stress is a significant risk factor for depression. Stress leads to peripheral and central immune activation; immune activation is associated with reduced dopamine (DA) neural function; and DA function underlies reward interest. A reduced interest in or inability to experience reward interest is a core symptom of depression. Research in mice demonstrated that Chronic Social Stress (CSS) mice exhibited higher spleen levels of granulocytes, inflammatory monocytes and $\mathrm{T}$ helper 17 cells; increased plasma levels of inducible nitric oxide synthase (iNOS); and liver expression of genes encoding kynurenine pathway enzymes, which are involved in vasodilation during inflammation and regulation of immune response $[49,50]$. In this research, the effect of CSS in the ventral tegmental area resulted in higher levels of kynurenine 
and the microglia markers Ibal and Cd11b and higher binding activity of DAD1 receptor; and in the Nucleus accumbens (NAcc) to higher kynurenine, lower DA turnover and lower c-Fos expression. CSS mice exhibited less reward-directed behavior. Therefore, this mouse study demonstrates that a chronic social stress leads to changes in each of the immune, neural and behavioral states proposed to mediate between stress and disruption of DA-dependent reward processing. The indication is therefore that boosting dopamine functional competence promotes greater stress tolerance and immune competence. Numerous studies demonstrate that a nutrigenomic dopamine agonist, KB220Z and variants, results in improved stress tolerance [39,51-53].

\section{Autoimmune disorders}

Autoimmune disorders are complex multifaceted pathophysiological disorders. While therapeutic interventions that target immune suppression and anti-inflammatory mechanisms can provide a degree of symptomatic management and relief, they are limited from addressing the broader systemic range of etiological factors, such as 1. errors in the synthesis of tissues requiring the appropriate perfect 3-dimentional structures for immunological acceptability; 2 . Inadequate neurotransmitter influences; 3 . Impaired hormonal influences, to name a few. Up until the writing of this paper, approximately 80 autoimmune disorders have been identified and explored, and the critical role of an aberrant immune response has been intricately associated with different organs and tissues. Hormonal homeostasis has great influence in achieving healthy and competent immune system function. Prolactin, which plays an essential role in metabolism, regulation of the immune system and pancreatic development, is secreted from the pituitary gland and biochemically acts as a hormone as well as a cytokine, which also prompts modulation of the immune system. Aberrant immune function is the dominant feature in patients suffering from diverse autoimmune diseases. However, upregulated 'autoimmune' attacks on the body's own tissues may have been misconceptualized. What appears to be an aberrant autoimmune attack could be a relentless and defensive upregulated immune response to imperfectly formed molecular structures being immunologically identified as antigens [54].

For example, hyperprolactinemia pushes immune responsivity and is evident in many autoimmune disorders including rheumatoid arthritis, systemic lupus erythematosus, Sjögren syndrome, multiple sclerosis, autoimmune thyroid disease and systemic sclerosis. Dopamine agonist therapy has been shown to exhibit benefits in autoimmune patients and offers a potential for therapeutic intervention with promising results [55].

\section{Optimizing dopamine homeostasis utilizing a patented gene test and a research validated nutrigenomic intervention}

Blum's group invested over 60 years of diligent devoted scientific research investigating the dose dependent inter-relationships of various nutraceutical ingredients and how they synergistically promote optimal gene expression and neurotransmitter 'cross-talk' between Serotonin, Enkephalin, Endorphin, GABA, Cannabinoids, and Dopamine, which he systemically termed the 'Brain Reward Cascade' (BRC). The research led to the development of the KB220 and subsequently the KB220Z nutrigenomic technology based on the dose-dependent relationships of ingredients that positively influenced gene expression in the BRC. The synergistic dose-dependent functional inter-relationships between the ingredients demonstrates that there is not one ingredient in the patented technology responsible for the expansive range of therapeutic effects on the BRC; it is the synergistic net effect of "The orchestra of nutrition playing the symphony of neurobiology".

The need to restore dopamine functionality by optimizing gene expression for all neurotransmitters in the BRC to reanimate neurotransmitter interconnectivity (i.e. 'cross talk') led Blum to develop the patented Genetic Addiction Risk Score (GARS) gene test for a DNADesigned Precision Nutrigenomic technology in the form of the next generation of the KB220Z PBM ('Precision Behavioral Management') [56-59]. In addition to the other benefits cited above regarding dopamine function, the KB220Z nutrigenomic technology has been shown in no less than 43 published clinical studies to reduce cravings and relapse [60], relieve stress, anxiety and nightmares [39,59,61]; enhance focus, concentration, cognition, and executive function; improve Blood Oxygen Level Dependent functioning [57,59,61,62], and elevate mood [63], among others. Consequently, other systemic beneficial effects cited above in this paper have not received the same notoriety owing to the societal magnitude of the opioid epidemic and the need for effective non-drug interventions. However, the purpose of this treatise is to provide greater mechanistic insight into the more expansive range of systemic benefits by coupling GARS and the Prodopamine regulator KB220Z PBM [64].

\section{Achieving happiness induces immunity:}

It is very interesting that older cultures such as Bhutan, believe that enlightenment through multiple paths, including Meditation, Yoga, and spiritual teachings, lead the way to satisfaction and fulfillment. In 1972 the then King of the country proclaimed that instead of measuring success by wealth or the "Gross National Product" it should be measured by "Gross National Happiness." Through many incarnations, one may become enlightened and reach the ultimate state of nirvana. Buddha described nirvana as the perfect peace of the state of mind that is free from craving, anger, and other afflictive states (Klesha) (The subject is at peace with the world, has compassion for all, and gives up obsessions and fixations). This peace is achieved when the existing volitional formations are pacified, and the conditions for the production of new ones are eradicated. In Nibbana, the root causes of craving and aversion have been extinguished such that one is no longer subject to human suffering (dukha) or further states of rebirths in (samsara).

With this in mind and being grateful for having this uplifting cultural mandate one could make a suggestion that NIRVANA is indeed an important acronym whereby it could be defined as "Neurotransmitter Interaction at Reward Ventral tegmental Accumbens leading to Neuronal Adaptation (NIRVANA)" Or Happiness. It is well-known that stress increases inflammatory agents while happiness reduces them. We are hereby proposing a novel model to help achieve better health during the Covid 19 pandemic (Figure 1).

Our view is that since many of aberrant behaviors are a consequence of and fall under RDS, one initial goal in the scientific pursuit of happiness in the human would be to first identify those gene polymorphisms associated with RDS and then utilize genomic principles to develop novel tailor-made pro-happiness agonists utilizing immunological compatible ('body friendly') natural substances. It is important to realize that everything that our brain does is ultimately the result of an interaction between our genes and our environment. For some traits, our genes play a major role, while for others, environment is a primary factor; for most, it is a combination of the two. On average, depending upon the trait, the genetic contribution accounts for 40 to 90 percent of a trait. In the latter case, it is a combination of many genes, 
Downs BW (2020) Molecular neuro-biological and systemic health benefits of achieving dopamine homeostasis in the face of a catastrophic pandemic (COVID- 19): A mechanistic exploration

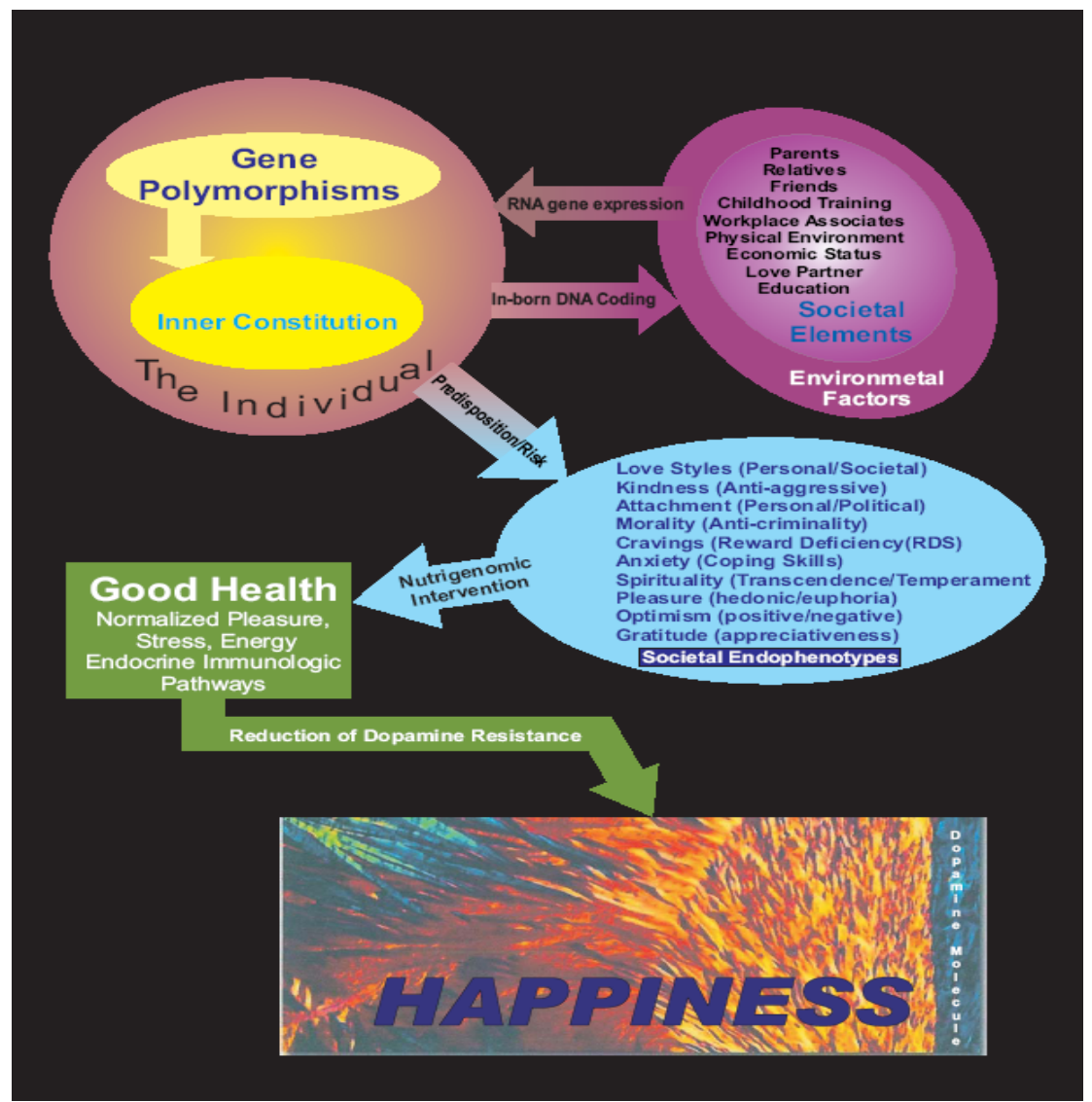

Figure 1. Happiness model: Schematic of genetic and environmental interactions leading to a reduction of "dopamine resistance" and a state of happiness [65]

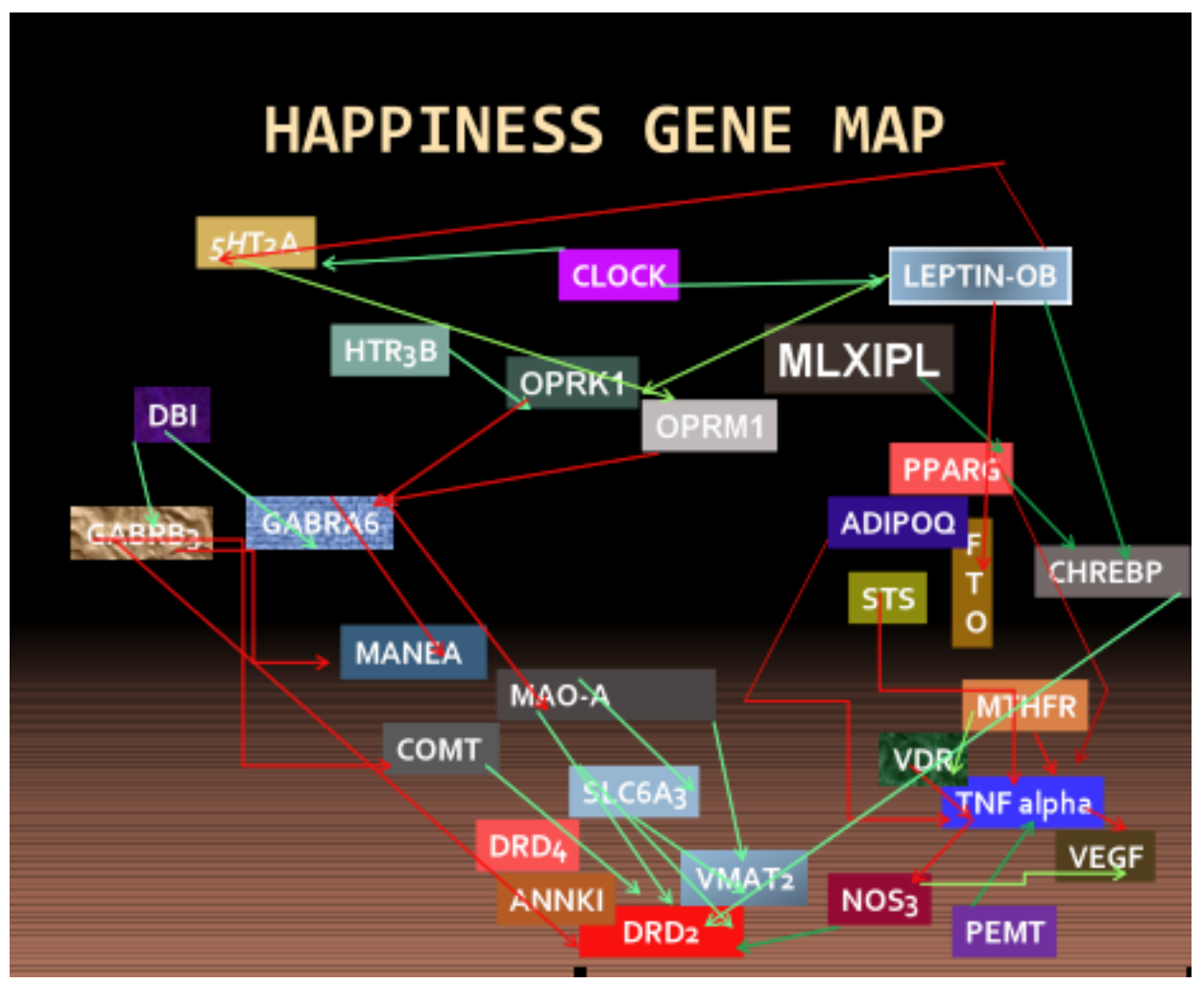

Figure 2. Proposed happiness gene map [65] 
Downs BW (2020) Molecular neuro-biological and systemic health benefits of achieving dopamine homeostasis in the face of a catastrophic pandemic (COVID- 19): A mechanistic exploration

referred to as "polygenic inheritance". To reiterate, it is well established that "polygenic inheritance" is due to the additive and epistatic interaction of many genes, each accounting for only a small percent of the total trait and interacting with the environment $[65,66]$ (Figure 2).

\section{Conclusion}

An extensive dossier of clinical and animal research indicates that in addition to promoting manageable reduction in addiction, obsession, compulsion, impulsion and an expansive range of aberrant personality disorders, achieving dopamine homeostasis with a number of epigenetic anti-dopaminylation insults possibly including a DNAdesigned precision nutrigenomic intervention (KB220Z PBM) provides evidenced based systemic benefits including: restoring optimal aerobic metabolism in the brain by improving Blood Oxygen Level Dependent signaling and functioning; reducing anaerobic pathologies and the generation of Reactive Oxygen Species; inducing neurotransmitter interconnectivity (aka 'crosstalk'); promoting restful sleep and waking accompanied by an increase in happy dreams; improving gut-brain signaling; reducing gastrointestinal inflammation and promoting GI tract functional competence; improving appetite regulation and food intake; improving vision and circadian rhythm regulation; and, importantly, reducing the effects of stress and improving immune competence $[9-23,30,33,34,52,53,58,60,63,64]$ This treatise expands our understanding of the health benefits of achieving dopamine homeostasis.

\section{Conflicts of interest}

Kenneth Blum, PhD assigned his IP to GARS IP, LLC., Hollywood Florida. Drs. Blum and Brewer are paid employees of GARS IP, LLC., and Geneus Health, LLC.

\section{Acknowledgements}

The authors appreciate the expert edits of Margaret A. Madigan.

\section{Author contribution}

All authors contributed equally.

\section{Funding source}

NIH grant R01NS073884 awarded to Rajendra D. Badgaiyan; Kenneth Blum and Marjorie C. Gondre-Lewis are recipients of R41 MD012318/MD/NIMHD NIH HHS/United States.

\section{References}

1. Center for Disease Control and Prevention (CDC). Coronavirus (COVID- 19) (https:// www.coronavirus.gov) (accessed Mar 25, 2020).

2. Velavan TP, Meyer CG (2020) The COVID-19 epidemic. Trop Med Int Health 25: 278-280.

3. Fischer B, Pang M, Jones W (2020) The opioid mortality epidemic in North America: do we understand the supply side dynamics of this unprecedented crisis? Subst Abuse Treat Prev Policy 15: 14.

4. Smoller JW (2016) The genetics of stress-related disorders: PTSD, depression, and anxiety disorders. Neuropsychopharmacology 41: 297-319.

5. Kranzler HR, Zhou H, Kember RL, Vickers Smith R, Justice AC, et al. (2019) Genomewide association study of alcohol consumption and use disorder in 274,424 individuals from multiple populations. Nat Commun 10: 1499.

6. Zilverstand A, Huang AS, Alia-Klein N, Goldstein RZ (2018) Neuroimaging impaired response inhibition and salience attribution in human drug addiction: A systematic review. Neuron 98: 886-903.
7. Zimmerman G, Shaltiel G, Barbash S, Cohen J, Gasho CJ, et al. (2012) Post-traumatic anxiety associates with failure of the innate immune receptor TLR9 to evade the proinflammatory NFאB pathway. Transl Psychiatry 2: e78.

8. Plein LM, Rittner HL (2018) Opioids and the immune system - friend or foe. $B r J$ Pharmacol 175: 2717-2725.

9. Blum K, Briggs AH, Trachtenberg MC, Delallo L, Wallace JE, et al. (1987) Enkephalinase inhibition: regulation of ethanol intake in genetically predisposed mice. Alcohol 4: 449-456.

10. Blum K, Wallace JE, Briggs AH, Trachtenberg MC (1985) Evidence for the importance of the "genotype" theory in alcohol seeking behavior: a commentary. Alcohol Drug Res 6: 455-461.

11. Janković BD, Radulović J (1992) Enkephalins, brain and immunity: modulation of immune responses by methionine-enkephalin injected into the cerebral cavity. Int $J$ Neurosci 67: 241-270.

12. Blum K, Chen ALC, Thanos PK (2018) Genetic addiction risk score (GARS) ${ }^{\mathrm{TM}}$, a predictor of vulnerability to opioid dependence. Front Biosci (Elite Ed) 10: 175-196.

13. Blum K, Modestino EJ, Neary J (2018) Promoting precision addiction management (PAM) to combat the global opioid crisis. Biomed J Sci Tech Res 2: 1-4.

14. Blum K, Madigan MA, Fried L, Braverman ER, Giordano J, et al. (2017) Coupling genetic addiction risk score (GARS) and pro dopamine regulation (KB220) to combat substance use disorder (SUD). Glob J Addict Rehabil Med 1: 555556.

15. Blum K, Modestino EJ, Gondre-Lewis M (2017) Dopamine homeostasis requires balanced polypharmacy: Issue with destructive, powerful dopamine agents to combat America's drug epidemic. J Syst Integr Neurosci 3: 1-6.

16. Downs BW, Blum K, Baron D (2019) Death by Opioids: Are there non-addictive scientific solutions? J Syst Integr Neurosci 5: 1-4

17. Fried L, Modestino EJ, Siwicki D, Lott L, Thanos PK, et al. (2019) Hypodopaminergia and "Precision Behavioral Management" (PBM): It is a generational family affair. Curr Pharm Biotechnol.

18. Blum K, Modestino EJ, Lott L (2018) Introducing "Precision Addiction Management $\left(\mathrm{PAM}^{\circledR}\right) "$ as an Adjunctive Genetic Guided Therapy for Abusable Drugs in America. Open Access J Behav Sci Psychol 1: 1-4.

19. Abijo T, Blum K, Gondre-Lewis MC (2019) Neuropharmacological and neurogenetic correlates of opioid use disorder (OUD) as a function of ethnicity: Relevance to precision addiction medicine. Curr Neuropharmacol.

20. Blum K, Gold M, Modestino EJ, Baron D, Boyett B, et al. (2018) Would induction of dopamine homeostasis via coupling genetic addiction risk score (GARS $\left.{ }^{\circledR}\right)$ and pro-dopamine regulation benefit benzodiazepine use disorder (BUD)? J Syst Integr Neurosci 4: 1-6

21. Blum K, Baron D, McLaughlin T, Gold MS (2020) Molecular neurological correlates of endorphinergic/dopaminergic mechanisms in reward circuitry linked to endorphinergic deficiency syndrome (EDS). J Neurol Sci 411: 116733.

22. Blum K, Modestino EJ, Gondré-Lewis MC, Neary J, Siwicki D, et al. (2017) Global opioid epidemic: doomed to fail without genetically based precision addiction medicine $\left(\right.$ Pam $\left.^{\mathrm{TM}}\right)$ : Lessons learned from America. Precis Med (Bangalore) 2: 17-22.

23. Blum K, Noble EP, Sheridan PJ, Montgomery A, Ritchie T, et al. (1990) Allelic association of human dopamine D2 receptor gene in alcoholism. JAMA 263: 20552060.

24. American society of addiction medicine (ASAM). Public policy statement; Definition of addiction, 2019, https://www.asam.org/resources/definition-of-addiction (Accessed Mar 15, 2020).

25. Lipner LM, Mendelsohn R, Muran JC (2017) Psychoanalysis. In: The SAGE encyclopedia of abnormal and clinical psychology. Ed. Wenzel A. SAGE Publications, Inc., Thousand Oaks, CA, pp. 2709-2711.

26. Oesterle TS, Thusius NJ, Rummans TA, Gold MS (2019) Medication-assisted treatment for opioid-use disorder. Mayo Clin Proc 94: 2072-2086.

27. National alliance on mental illness. NAMI Hernando. https://namihernando.org/ (accessed Mar 16, 2020).

28. Koehl JL, Zimmerman DE, Bridgeman PJ (2019) Medications for management of opioid use disorder. Am J Health Syst Pharm 76: 1097-1103. 
Downs BW (2020) Molecular neuro-biological and systemic health benefits of achieving dopamine homeostasis in the face of a catastrophic pandemic (COVID- 19): A mechanistic exploration

29. Blum K, Thompson B, Demotrovics Z, Femino J, Giordano J, et al. (2015) The molecular neurobiology of twelve steps program \& fellowship: Connecting the dots for recovery. J Reward Defic Syndr 1: 46-64.

30. Corbier JR, Downs BW, Kushner S, Aloisio T, Bagchi D, et al. (2019) VMP35 MNC, a novel iron-free supplement, enhances cytoprotection against anemia in human subjects: a novel hypothesis. Food Nutr Res 63: 3410 .

31. Bagchi D, Garg A, Krohn RL, Bagchi M, Bagchi DJ, et al. (1998) Protective effects of grape seed proanthocyanidins and selected antioxidants against TPA-induced hepatic and brain lipid peroxidation and DNA fragmentation, and peritoneal macrophage activation in mice. Gen Pharmacol 19930: 771-776.

32. Downs BW, Bagchi M(2017)A hidden etiological nemesis of chronic neurodegenerative diseases. In: Phytopharmaceuticals for brain health. Eds: Subhan S, Bagchi M. CRC Press/Taylor \& Francis Group, Boca Raton, FL, Chapter 1: 3-16.

33. Febo M, Blum K, Badgaiyan RD, Perez PD, Colon-Perez LM, et al. (2017) Enhanced functional connectivity and volume between cognitive and reward centers of naïve rodent brain produced by pro-dopaminergic agent KB220Z. PLoS One 12: e0174774.

34. Blum K, Liu Y, Wang W, Wang Y, Zhang Y, et al. (2015) rsfMRI effects of KB220ZTM on neural pathways in reward circuitry of abstinent genotyped heroin addicts. Postgrad Med 127: 232-241.

35. Qu WM, Xu XH, Yan MM, Wang YQ, Urade Y, et al. (2010) Essential role of dopamine D2 receptor in the maintenance of wakefulness, but not in homeostatic regulation of sleep, in mice. $J$ Neurosci 30: 4382-4389.

36. Monti JM, Jantos H (2008) The roles of dopamine and serotonin, and of their receptors, in regulating sleep and waking. Prog Brain Res 172: 625-646.

37. Blum K, Oscar-Berman M, Badgaiyan RD, Khurshid KA, Gold MS, et al. (2014) Dopaminergic neurogenetics of sleep disorders in reward deficiency syndrome (RDS). J Sleep Disord Ther 3: 126-132.

38. Xie L, Kang H, Xu Q, Chen MJ, Liao Y, et al. (2013) Sleep drives metabolite clearance from the adult brain. Science 342: 373-377.

39. McLaughlin T, Febo M, Badgaiyan RD, Barh D, Dushaj K, et al. (2016) K. KB220ZTM a pro-dopamine regulator associated with the protracted, alleviation of terrifying lucid dreams. Can we infer neuroplasticity-induced changes in the reward circuit? J Reward Defic Syndr Addict Sci 2: 3-13

40. Landolt HP (2011) Genetic determination of sleep EEG profiles in healthy humans Prog Brain Res 193: 51-61.

41. Holst SC, Bersagliere A, Bachmann V, Berger W, Achermann P, et al. (2014) Dopaminergic role in regulating neurophysiological markers of sleep homeostasis in humans. J Neurosci 4: $566-573$.

42. Pieroni MA (2019) Investigation of apomorphine during sleep in Parkinson's improvement in UPDRS Scores. Neurol Int 11: 8207.

43. Garrido-Gil P, Rodriguez-Perez AI, Dominguez-Meijide A, Guerra MJ, LabandeiraGarcia JL, et al. (2018) Bidirectional neural interaction between central dopaminergic and gut lesions in parkinson's disease models. Mol Neurobiol 55: 7297-7316.

44. Downs BW, Corbier JR, Speight N, Kushner S, Aloisio T, et al. (2020) Anemia: Influence of dietary fat, sugar, and salt on hemoglobin and blood health. In: Dietary sugar, salt, and fat in human health. In: Dietary sugar, salt, and fat in human health.

45. Sclafani A, Ackroff K (2012) Role of gut nutrient sensing in stimulating appetite and conditioning food preferences. Am J Physiol Regul Integr Comp Physiol 302: 1119-1133.

46. Smith BJ, Cote PD, Tremblay F (2015) Dopamine modulation of rod pathway signaling by suppression of GABAC feedback to rod-driven depolarizing bipolar cells. Eur $J$ Neurosci 42: 2258-2270.

47. Felder-Schmittbuhl MP, Buhr ED, Dkhissi-Benyaha O, Hicks D, Pierson SN, et al. (2018) Ocular clocks: Adapting mechanisms for eye functions and health. Invest Opthalmol Vis Sci 59: 4856-4870.
48. Blum K, Oscar-Berman M, Badgaiyan R, Braverman ER, Gold MS, et al. (2014) Hypothesizing darkness induced alcohol intake linked to dopaminergic regulation of brain function. Psychology (Irvine) 5: 282-288.

49. Bergamini G, Mechtersheimer J, Azzinnari D, Sigirst H, Buerge M, et al. (2018) Chronic social stress induces peripheral and central immune activation, blunted mesolimbic dopamine function, and reduced reward-directed behaviour in mice. Neurobiol Stress 8: 42-56.

50. Wang Y, Liu H, McKenzie G, Witting PK, Stasch JP, et al. (2010) Kynurenine is an endothelium-derived relaxing factor produced during inflammation. (2010) Nature Medicine 16: 279-285.

51. Madrid GA, MacMurray J, Lee JW, Anderson BA, Comings DE, et al. (2001) Stress as a mediating factor in the association between the DRD2 TaqI polymorphism and alcoholism. Alcohol 23: 117-122.

52. Solanki N, Abijo T, Galvao C, Darius P, Blum K, et al. (2020) Administration of a putative pro-dopamine regulator, a neuronutrient, mitigates alcohol intake in alcoholpreferring rats. Behavioral Brain Res 385: 112563.

53. Blum K, Trachtenberg MC, Elliott CE, Dingler ML, Sexton RL, et al. (1988) Enkephalinase inhibition and precursor amino acid loading improves inpatien treatment of alcohol and polydrug abusers: double-blind placebo-controlled study of the nutritional adjunct SAAVE. Alcohol 5: 481-493.

54. Bagchi D, Misner B, Bagchi M, Kothari SC, Downs BW, et al. (2002) Effects of orally administered undenatured type II collagen against arthritic inflammatory diseases: A mechanistic exploration. Intl J Clin Pharmacol Res 22: 101-110.

55. Borba VV, Zandman-Goddard G, Shoenfeld Y (2019) Prolactin and autoimmunity: The hormone as an inflammatory cytokine. Best Pract Res Clin Endocrinol Metab 11 101324.

56. Blum K, Oscar-Berman M, Demetrovics Z, Barh D, Gold MS, et al. (2014) genetic addiction risk score (GARS): Molecular neurogenetic evidence for predisposition to reward deficiency syndrome (RDS). Molecular Neurobiology 50: 765-796.

57. Blum K, Giordano J, Morse S, Liu Y, Tan J, et al. (2010) Research: Pharmacogenomic genetic addiction risk score (GARS) analysis: Exploratory development of polymorphic risk alleles in poly-drug addicted males. The IIOAB 1: 1-14

58. Blum K, Oscar-Berman M, DiNubile N, Giordano J, Braverman ER, et al. (2013) Coupling genetic addiction risk score (GARS) with electrotherapy: Fighting iatrogenic opioid dependence. J Addict Res Ther 4: 1000163.

59. Blum K, Chen ALC, Thanos PK, Febo M, Demetrovics Z, et al. (2018) Genetic addiction risk score (GARS) ${ }^{\mathrm{TM}}$, a predictor of vulnerability to opioid dependence. Front Biosci (Elite Ed) 10: 175-196.

60. Downs BW, Blum K, Baron D, Bowirrat A, Lott L, et al. (2019) Death by opiods: Are there non-addictive scientific solutions? J Syst Integr Neurosci 5: 1-4.

61. Shorrock HK, van der Hoorn D, Boyd PJ, Liavero-Hurtado M, Lamont DJ, et al. (2018) UBA1/GARS-dependent pathways drive sensory-motor connectivity defects in spinal muscular atrophy. Brain 141: 2878-2894.

62. Blum K, Baron D, McLaughlin T, Gold MS (2020) Molecular neurological correlates of endorphinergic/dopaminergic mechanisms in reward circuitry linked to endorphinergic deficiency syndrome (EDS). J Neurol Sci 411: 116733.

63. Blum K, Siwicki D, Baron D, Modestino EJ, Badgaiyan RD, et al. (2018) The benefit of genetic addiction risk score $\left(\mathrm{GARS}^{\mathrm{TM}}\right)$ and pro-dopamine regulation in combating suicide in the American Indian population. $J$ Syst Integr Neurosci 4: 1-6

64. Blum K, Gondré-Lewis MC, Baron D, Thanos PK, Braverman ER, et al. (2018) Introducing precision addiction management of reward deficiency syndrome, the construct that underpins all addictive behaviors. Front Psychiatry 9: 548.

65. Blum K (2009) Genes \& happiness. Gene Ther Mol Biol 13

66. Stern C (1973) Principles of human genetics. W.H. Freedman, San Fransico.

Copyright: (C2020 Downs BW. This is an open-access article distributed under the terms of the Creative Commons Attribution License, which permits unrestricted use, distribution, and reproduction in any medium, provided the original author and source are credited. 